\title{
Autoimmune Hemolytic Anemia in Intensive Care Unit and Blood Transfusion: Lesson Learnt_-A Case Report
}

\author{
Sunil K Garg ${ }^{1}$, Pragya Garg²®
}

\begin{abstract}
Clinicians are often in a difficult situation while managing severe anemia due to autoimmune hemolysis in intensive care unit (ICU). It is hard to get properly cross-matched blood due to the presence of autoantibody in the patient's serum. Still, such patients should not be devoid of transfusion.

Keywords: Antiglobulin test, Autoantibodies, Autoimmune hemolytic anemia, Cross-matching incompatibility.

Indian Journal of Critical Care Medicine (2021): 10.5005/jp-journals-10071-23999
\end{abstract}

\section{INTRODUCTION}

Autoimmune hemolytic anemia (AIHA) is a type of acquired hemolytic anemia because of development of antibodies against antigen on a patient's own erythrocytes that lead to increased destruction of erythrocytes. ${ }^{1}$ It takes place with an incidence in adults of $0.8-3$ per $10^{5} /$ year, a prevalence of $17: 100,000$, and a mortality rate of $11 \% .2,3$ Major causes include idiopathic (50\%), autoimmune diseases (20\%), lymphoproliferative syndromes (20\%), tumors, and infections. ${ }^{4}$

\section{Case Description}

An 80-year-old male with a history of benign prostatic hypertrophy presented with the discoloration of urine to a urologist. Thereafter, he was referred to a physician in view of increased urinary urobilinogen. In view of increased urinary urobilinogen and absent urinary bilirubin, suspicion of hemolysis was made. On further detailed history, he mentioned weakness, tiredness, fatigability, and the shortness of breath for the last few days. He also mentioned slight weakness and low appetite for the past few months and underwent coronary angiography for the same which came out to be normal. On admission, patient was conscious, with mild tachycardia, tachypnea, and temperature of $38.5^{\circ} \mathrm{C}$. His examination was notable for the presence of pallor and icterus.

On initial evaluation in out-patient department (OPD), complete blood count showed a high white blood cell count of 23,500 with neutrophil count $64.9 \%$, hemoglobin $(\mathrm{Hb}) 8.1 \mathrm{~g} / \mathrm{dL}$, mean corpuscular volume $104 \mathrm{fL}$, and platelets 313,000 . On the day of admission, 2 days later, his $\mathrm{Hb}$ further dropped to $5.3 \mathrm{~g} / \mathrm{dL}$. Further workup revealed aspartate aminotransferase (AST) $85 \mathrm{U} / \mathrm{L}$, alanine aminotransferase (ALT) $30 \mathrm{U} / \mathrm{L}$, serum albumin $4.2 \mathrm{~g} / \mathrm{dL}$ serum ferritin $1348.4 \mathrm{ng} / \mathrm{dL}$, Indirect bilirubin $5.1 \mathrm{mg} / \mathrm{dL}$, direct bilirubin $0.8 \mathrm{mg} /$ $\mathrm{dL}$, lactate dehydrogenase $1481 \mathrm{IU} / \mathrm{L}$, and normal coagulation profile. His reticulocyte count was $15 \%$. Peripheral smear showed leukoerythroblastic picture with features of hemolytic anemia. Both direct and indirect Coombs' tests were positive.

The ultrasonography (USG) abdomen was positive only for minimal coarse echotexture of liver while spleen size and echotexture were normal. X-ray chest showed minimal haziness in the left lower zone. His screening for HIV, HBsAg, HCV, and
${ }^{1,2}$ Department of Critical Care, NMC Healthcare, Dubai, United Arab Emirates

Corresponding Author: Sunil K Garg, Department of Critical Care, NMC Healthcare, Dubai, United Arab Emirates, Phone: +91-11-22334455, e-mail: sucare12@yahoo.co.in

How to cite this article: Garg SK, Garg P. Autoimmune Hemolytic Anemia in Intensive Care Unit and Blood Transfusion: Lesson Learnt-A Case Report. Indian J Crit Care Med 2021;25(10):1201-1202.

Source of support: Nil

Conflict of interest: None

malaria was negative. Further workup including characterization of antibody was sent. There was no prior history of blood transfusion. There was no evidence of lymphoma or any other malignancies. He was negative for mycoplasma serology.

He was started on prednisolone and antibiotic. In view of drop in $\mathrm{Hb}$ to $5.2 \mathrm{~g} / \mathrm{dL}$, the request was made to the blood bank for arranging group-specific blood. Around 12 hours after admission, the patient developed an increasing heart rate which continued to worsen over the next few hours. Blood gas analysis done at this time revealed metabolic acidosis and further fall in hematocrit. Request for blood transfusion was sent again for the group-specific blood but the blood bank did not release the blood and the patient could not be transfused since all available units were incompatible. Finally, the patient succumbed to his illness in less than 24 hours of hospital admission.

\section{Discussion}

Autoimmune hemolytic anemia is an infrequent disorder manifested by autoantibody-induced hemolysis against own red blood cells (RBCs). It is mainly divided as warm AlHA which is due to warm-reactive lgG-mediated extravascular hemolysis, consisting $75 \%$ of all patients, and cold AlHA which occurs mainly because of complement-mediated hemolysis in intravascular compartment, and it comprises about $15 \%$ of total AlHA patients. Rest of the cases which are less than $5 \%$ are mixed type.

This division is based on the temperature range of autoantibodies implicated in pathogenesis. The laboratory diagnosis of AlHA is 
based on the result of direct Coombs' test (DAT). This test shows reactivity with anti-IgG (usually in warm type) and/or anti-C3d (usually in cold type) antisera and the presence of findings suggesting hemolysis. However, since direct Coombs' test may produce false-negative results in up to $10 \%$ of all patients, the patient may have DAT-negative AIHA.

There is a general agreement that corticosteroids are the most effective treatment for patients with warm type, with a response rate of $70-85 \%$. The splenectomy is considered effective second-line therapy, providing the initial response of up to $82 \%$ in patients with warm type. Rituximab is also an effective second-line treatment, with an initial response rate of up to $87 \%$.

Transfusion of patients with AlHA presents with a unique set of potential problems. ${ }^{5}$ Transfusion is of temporary benefit but essential when anemia is severe. This process of RBC transfusion in this disorder can be tricky because of cross-matching problems as it happened in our case and rapid destruction of transfused RBCs occurs due to the presence of the auto- or alloantibody.

Clinicians are often in a difficult situation and reluctant to transfuse incompatible blood when such a situation arises. A discussion between clinician and transfusion specialist should take place as soon as the patient of AlHA is admitted to the intensive care unit (ICU). After appropriate evaluation and testing, the clinician should be made confident that transfusion will be unlikely to cause an acute hemolytic reaction. However, it is likely that RBCs will not survive normally. ${ }^{5}$ At our center, transfusion medicine specialist services were not available.

Our patient had a broadly reactive autoantibody and the transfusion service found that all units of RBCs were incompatible; therefore, they could not release blood for transfusion at the time of exigency and this is a frequently encountered mistake during transfusion of such patients. There is disinclination for the transfusion in such patients due to unpredictability regarding safety and efficacy of blood that is noncompatible because of the presence of an RBC autoantibody. In such patients, hemolytic transfusion reactions occur when alloantibodies are present. ${ }^{5}$ When the RBC unit is not compatible because of RBC autoantibody only, the transfused RBC survival is mostly as good as normal, and transfusion is likely to provide substantial temporary benefit. ${ }^{5}$ If compatibility testing is done adequately and appropriately to detect RBC alloantibody, the indications of RBC transfusions are not much different in patients with this disorder than for similarly anemic patients without AIHA. ${ }^{5}$ Examples of patients who were not given transfusion despite clear indications have been reported by Conley et al. ${ }^{6}$ They reported five patients with this disorder who manifested with life-threatening anemia who was denied transfusion since their caregivers were concerned that available blood is not compatible.

Only a minority of all hospitalized have alloantibody so if transfusion is deemed necessary, it is appropriate to transfuse the patient as it entails a lesser risk than waiting for compatibility testing. ${ }^{5}$ Probability of having alloantibody in a person is very low who has no history of previous transfusion or pregnancy. Due to the absence of these factors in our patient, he was at very low risk of having alloantibody. So, if transfusion is extremely urgent, as in our patient, it should not be withheld even in the absence of compatible blood.

\section{Conclusion}

It is very essential to comprehend the basics and importance of compatibility test method in patients with AlHA and to activate transfusion services as soon as AIHA patient arrives in ICU or is diagnosed to be AlHA. Clinicians and transfusion medicine specialists both must act proactively and plan so any exigency can be managed effectively, since the patient cannot be devoid of transfusion due to blood incompatibility.

\section{OrCID}

Sunil K Garg @ https://orcid.org/0000-0001-9710-8736

Pragya Garg (1) https://orcid.org/0000-0003-4673-5910

\section{References}

1. GC Gruchy. The haemolytic anaemias. De Gruchy's clinical hematology in medical practice. Blackwell Science Ltd; 1989. p. $172-175$.

2. Petz LD, Garratty G. Immune haemolytic anemias. 2nd ed. Philadelphia: Churchill Livingstone; 2014.

3. Lechner K, Jager U. How I treat autoimmune haemolytic anemias in adults. Blood 2010;116(11):1831-1838. DOI: 10.1182/blood-2010-03259325.

4. Valent $P$, Lechner K. Diagnosis and treatment of autoimmune haemolytic anaemias in adults: a clinical review. Wien Klin Wochenschr 2008;120(5-6):136-151. DOI: 10.1007/s00508-008-0945-1.

5. Lawrence D, Petz MD. A physician's guide to transfusion in autoimmune hemolytic anaemia. Br J Hematol 2004;124(6):712-716. DOI: 10.1111/j.1365-2141.2004.04841.x.

6. Conley CL, Lippman SM, Ness P. Autoimmune hemolytic anaemia with reticulocytopenia. A medical emergency. JAMA 1980;244:1688-1690. DOI: 10.1056/NEJM198202043060507. 